\title{
Stereotaxic display of brain lesions
}

\author{
Chris Rorden ${ }^{\mathrm{a}, *}$ and Matthew Brett ${ }^{\mathrm{b}}$ \\ ${ }^{a}$ University of Nottingham, Nottingham, UK \\ ${ }^{\mathrm{b}}$ MRC Cognition and Brain Sciences Unit, \\ Cambridge, $U K$
}

Traditionally lesion location has been reported using standard templates, text based descriptions or representative raw slices from the patient's CT or MRI scan. Each of these methods has drawbacks for the display of neuroanatomical data. One solution is to display MRI scans in the same stereotaxic space popular with researchers working in functional neuroimaging. Presenting brains in this format is useful as the slices correspond to the standard anatomical atlases used by neuroimagers. In addition, lesion position and volume are directly comparable across patients. This article describes freely available software for presenting stereotaxically aligned patient scans. This article focuses on MRI scans, but many of these tools are also applicable to other modalities (e.g. CT, PET and SPECT). We suggest that this technique of presenting lesions in terms of images normalized to standard stereotaxic space should become the standard for neuropsychological studies.

\section{Introduction}

Accurate lesion localization is vital in order to make anatomical inferences based on neuropsychological data. This article has three main aims. First, some of the limitations of the traditional methods for presenting lesion information will be discussed. Second, an alternative method (called 'spatial normalization') will be outlined. Normalization scales, rotates and warps the patient's Magnetic Resonance Imaging (MRI) scan so that it is approximately co-registered with a standard atlas template image. The advantages of this technique will be introduced. Third, the technical difficulties that have prevented this technique from gaining widespread acceptance will be reviewed. Finally, this article will

*Corresponding author: Chris Rorden, School of Psychology, University of Nottingham, University Park, Nottingham, NG7 2RD, UK. Tel.: +44 115951 5294; Fax: +44 115951 5324; E-mail: chris.rorden@nottingham.ac.uk. describe a number of recent advances that have generally overcome these difficulties.

Advances in neuroimaging such as functional MRI (fMRI) allow scientists to observe brain function in the intact human brain. These studies avoid some of the inherent limitations of neuropsychology. For example, the interpretation of most neuropsychological functions relies on the assumption that the preserved regions of a neurological patient's brain are functioning normally. This assumption is often wrong for two reasons. First, the brain is a highly integrated organ, and damage to one area will therefore have knock-on effects on the remaining regions. While clinical MRI scans can identify regions that are clearly damaged, this does not necessarily mean that the other regions are functioning normally (functional and diffusion-weighted imaging of neurological patients [14,19] can help address this problem). Secondly, brain function is often plastic, and therefore the function of preserved regions may adapt to compensate for focal damage.

However, neuropsychology also has intrinsic strengths. For example, the power of inference based on a conventional fMRI study is fairly weak. Generally, fMRI studies indicate that certain regions are involved with specific tasks. Neuropsychology can make a stronger claim, identifying required regions for certain tasks.

The inherent strengths of the neuropsychological method means it can play an important role in the emerging field of cognitive neuroscience. It can complement the use of other tools such as fMRI, single cell recording, positron emission tomography (PET), event related potentials (ERP), optical imaging and transcranial magnetic stimulation (TMS). Indeed, neuropsychology will perhaps become most useful as a tool for steering further exploration with other techniques. For example, patients with posterior cerebral artery territory infarcts often fail to recognize faces [4]; convergent findings using fMRI in healthy subjects show activation in this region when viewing faces [9]. The specific deficits found in patients can imply some level of modular processing, and suggest necessary regions for completing these tasks. Therefore, neuropsychology can guide the choice of task and anatomical location for research with tools such as TMS, fMRI and ERP. 
If neuropsychology is to make an important contribution to the emerging field of cognitive neuroscience, close attention must be paid to the specific nature and location of the lesion giving rise to the functional deficits. The next section reviews the limitations of the lesion-mapping techniques that are currently popular. Later sections describe an alternative method for presenting patient data.

\section{Traditional methods for presenting anatomical data}

There are three common methods for presenting anatomical data from brain-injured patients. One solution is to show the lesion on a template of a standard healthy brain. A second technique is to describe the lesion using only a text description. A final method is to display a representative slice or slices from the patient's clinical CT or MRI scan. This section will describe each of these techniques in turn, with an emphasis on the limitations of each.

Lesions are often displayed on a series of template brain slices [3,7] (see Fig. 1(A)). The advantage of this technique is that lesion location and volume from different patients can be roughly compared (even though actual brain size and orientation are different in the original scans). Presenting lesions on these universal templates helps the reader visualize the approximate location of the lesion. However, this method has a number of distinct drawbacks. First of all, standard neuropsychological CT-scan templates are distinctly different from the templates commonly used to display fMRI data. For example, compare Fig. 1(A) (a common neuropsychology template) with Fig. 4 (an image aligned to a template used by many neuroimagers). Note that pitch of the Fig. 1(A)'s slices match the common CT scanning angle, while the slice in Fig. 4 is aligned to easily recognized landmarks (as will be described later). Therefore, it is difficult to make comparisons between the patient data and functional imaging data. One of the biggest drawbacks of this technique is that the person drawing the lesion on the template needs to find the corresponding location on the clinical scan and manually define the lesion. This is difficult because the template and the scan will be viewed at different orientations and scales. Every brain has a unique pattern of sulci and gyri, and the person defining the lesion must determine how to translate the location of the lesion to a template of a generic brain. Therefore, this technique requires a skilled individual who must painstakingly make a number of difficult and often arbitrary decisions. Replication is difficult, as different individuals will focus on different landmarks. Another difficulty with this technique is that the final presentation is on a 'universal' brain, which can hide any unique variations found in the original lesion or the patient's brain. For example, the standard templates will hide dilated ventricles, mass effects (shifts in the location of brain structures due to edema, resorption, or tumors) or unique sulcal patterns.

A second technique for reporting lesion location is to present a text-based clinical description. This format is often vague and it can be difficult to determine the locus of the lesion with much accuracy based solely on a text-based report. In addition, lesion volume cannot be accurately compared across patients. Furthermore, precise comparisons with neuroimaging studies cannot be made. The readers are not able to inspect the scans themselves and make their own judgements.

The third common technique for presenting patient data is to present representative slice(s) of the patient's raw MRI or CT scan (as shown in Fig. 1(B)). This method preserves the morphological variations between patients. However, the burden of interpreting the lesion location and volume is placed on the reader. Because scans vary in scan angle and overall brain size, the slices do not correspond with neuroanatomical atlases. Therefore, it can be difficult for the reader to determine the location of a lesion. Furthermore, it is difficult to compare the size and location of lesions across patients. Again, this method makes it difficult to make comparisons between patients or with published neuroimaging studies.

In review, each of the contemporary methods for presenting lesion anatomy have limitations. Therefore, it is often difficult to relate neuropsychological findings to other methods. One solution is to present patient scans using the same stereotaxic techniques used by neuroimagers, as discussed in the next section. This approach can improve our understanding of the anatomical basis for neuropsychological findings.

\section{Normalized scans: an alternative way to present lesions}

An alternative technique for presenting anatomical information is to present the patient's own MRI scan after it has been coregistered to the same coordinate system used to present neuroimaging data. This technique of scaling, rotating and warping a brain to be aligned 


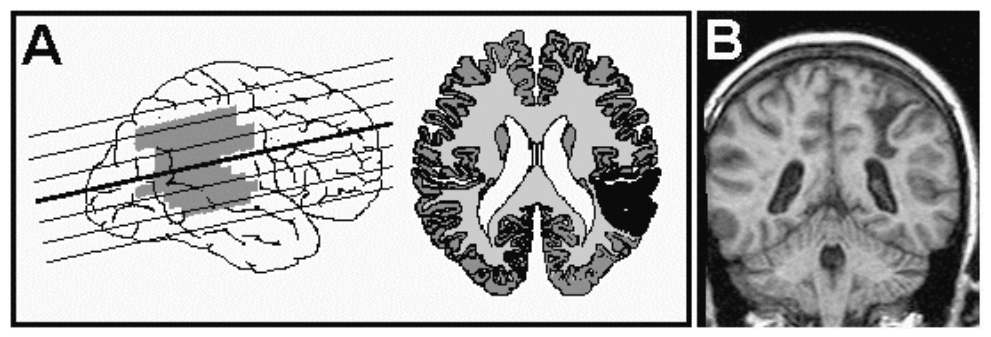

Fig. 1. Classic presentation of lesion location. A. Shows a lesion plotted on standard CT templates. Note the angle of the slices differs from the Talairach stereotaxic space favored by neuroimagers (see Fig. 4). B. A single representative MRI scan slice. This view shows the lesion location and ventricle size. However, this view does not correspond with any standard atlases, making it difficult to compare lesion location or volume across patients. Please note that images A and B come from different patients.

with an iconic template scan is generally referred to as 'spatial normalization'. Once an image is normalized, a series of slices can be presented that correspond with published atlases, neuroimaging studies and other neuropsychological studies where the patient scans have been similarly normalized.

This approach has five clear advantages over the techniques currently popular in neuropsychology. First of all, the lesion location is easier to identify, as readers can inspect the scan and a corresponding slice from an atlas. Secondly, normalized lesion volume is more meaningful, as the overall size of the brain has been normalized. Third, it is easier to compare lesion location across patients, as equivalent slices can be presented for each patient. Likewise, it is easier to draw comparisons with patient lesion location and published neuroimaging studies that have aligned their scans to the same template image. Fourthly, the patient's own scan is presented - so the readers can find relevant landmarks and unique features of the patient's brain. Finally, contemporary normalization is fairly automated, so it is not as technically demanding, time consuming, and subjective as hand drawn lesions on standard templates.

Normalization is a computationally intensive process and a great deal of research has gone into refining this technique. However, the basic concepts and terms are straightforward. In addition, modern implementations are fairly automatic and generally easy to use. The specific implementation used by SPM99 [1,8] will be described here (this software can be downloaded from www.fil.ion.ucl.ac.uk/spm/). There are other normalization packages that use similar intensity-based algorithms, such as AIR [20] and MNI_AutoReg/ANIMAL [2]. It should be noted that there are techniques that use radically different algorithms [17] that are preferable in certain applications. However, SPM's popularity with neuroimagers, robust- ness, and ability to normalize images with focal lesions (as discussed later) make it an appropriate example for a discussion of spatial normalization.

Normalization is an iterative process for matching an individual's brain image (the 'source') with a standard image (the 'template'). The software proceeds by trying a starting spatial transformation, and estimating how well the images are matched, using a 'cost function'. The cost function is a measure of how well the images are matched, and is low when the images are well matched and high when the match is poor. In SPM the cost function is the squared intensity difference between the images; in other words, the cost function will be low if the bright areas of the source are matched with bright areas of the template. Using a standard optimization algorithm [1], the software can then estimate the best spatial transformation, and then apply this, and recalculate the cost function. The program continues this process of successive adjustments and recalculation until the cost function is minimized.

To obtain an overall match, The SPM algorithm proceeds in stages. The source image is first spatially smoothed (in fact with a Gaussian filter of $8 \mathrm{~mm}$ full width at half maximum). This has the effect of attenuating the very fine pixel-to-pixel detail of the image, while emphasizing the overall brain shape. The template image has been similarly smoothed. Next the source and template images are matched, using affine spatial transformations such as rotations and zooms. The affine transformations are illustrated in Fig. 2, and apply across the whole image, and are therefore relatively robust to the effect of lesions in the source image (see below). When the optimum affine transformations have been calculated, so that the two images are in rough alignment, the skull and scalp are removed from the calculation of the cost function, using a masking image (see below). The affine parameters are then re-estimated, so that the match is optimized for brain 

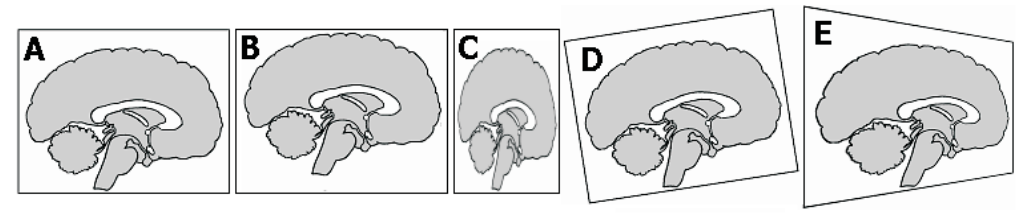

Fig. 2. An illustration of the common affine transformations. A. represents the standard template for this example. B. An image that must requires a translation (here, moved ventrally) in order to match the template. C. Shows an image that requires a 'zoom'. D. Illustrates an image that would require a 'rotation' (in this case, of pitch). E. Requires a 'shear' (aka affine) transformation. Each of these illustrative caricatures shows the specific transformation in only one of the three dimensions. Note that each of these transformations can be applied in each of the three dimensions (e.g. rotation can influence the pitch, yaw and roll of an image). Affine transformations are sometimes referred to as linear transformations. Note that normalization of most images requires some degree of each of these functions.

shape only. After the affine parameters have been optimized, the algorithm proceeds to calculate the optimum parameters for nonlinear transformations in the same iterative fashion as for the affine transforms. In SPM, the nonlinear functions are drawn from a discrete cosine transform basis set, which are a set of cosine wave functions that will selectively compress some portions of the image while expanding other regions [1]. Nonlinear functions improve the correspondence of the source image to the template [1], but they tend to be more sensitive to local differences than affine transforms. Because the non-linear transforms can have large effects that are quite local in the image, the nonlinear transforms can have a significant impact on the shape of the lesioned area of the patient's scan. The non-linear transforms are driven to change the shape of the lesioned area because the lesion will usually have a very different intensity in the patient's scan to the corresponding area in the template, and will thus contribute a large amount to the cost function that is to be minimized. This issue will be discussed later.

Neuroimagers typically normalize their images with template images developed by the Montreal Neurological Institute, and subsequently adopted by the International Consortium of Brain Mapping (ICBM) [5]. This template attempts to define a standard, by taking the average of the MRI brain scans from a large number of young normal individuals. Normalizing the MRI scans from neurological patients (particularly those with focal brain lesions) to this popular template maximizes the correspondence of the resulting images with published neuroimaging studies. However, when normalizing images from a group of patients with diffuse brain damage (e.g. Alzheimer's patients), it may be preferable to create a template based on the diseased group [17]. The 'Talairach' coordinate system [16] is typically used to describe spatial locations in these scans. ${ }^{1}$ While this coordinate system is unfamiliar to many neuropsychologists, it is fairly easy to describe (in a slightly simplified manner). First of all, Talairach coordinates are measured with respect to the anterior commissure (AC), a fibre tract that connects the two hemispheres that lies anterior to the thalamus. Three axes define the Talairach coordinate system: leftright (X-axis), posterior-anterior (Y-axis), and inferiorsuperior (Z-axis). All the axes pass through the AC: the Y-axis passes through both the anterior and posterior commissures; the Z-axis runs through the AC and up along the interhemispheric fissure, and the $\mathrm{X}$-axis runs perpendicular to the $\mathrm{Z}$ and $\mathrm{Y}$-axes. Thus the $\mathrm{AC}$ is defined as having a Talairach coordinate of $0,0,0$, and the standard axial plane of the Talairach system contains the $\mathrm{AC}$, and is the plane where the $\mathrm{Z}$ coordinate is zero (see Fig. 4). It should be mentioned that the orientation of this plane is quite different from the orientation of axial planes found in most CT scans (compare the slice orientation in Fig. 1(A) to the image in Fig. 4). Any point in the brain can be identified by its coordinate in millimetres relative to these $\mathrm{X}, \mathrm{Y}$ and $\mathrm{Z}$ axes, where a positive $\mathrm{X}$ coordinate is on the right of the $\mathrm{AC}$, positive $\mathrm{Y}$ is anterior to the $\mathrm{AC}$, and positive $\mathrm{Z}$ is above the $\mathrm{AC}$. For example, a point at coordinate $38,-64,58$ is in the right, posterior, superior portion of the brain.

Two features can be clearly seen from the sample normalized patient scans presented in Fig. 3. First of all, despite the large lesions, all of the images are fairly

\footnotetext{
${ }^{1}$ Neuroimagers use the coordinate system developed by Talairach and Tornoux [16]. Unfortunately, no MRI scans exist for the brain used to create the Talairach atlas, and therefore the MRI templates that are available vary slightly from the atlas. Therefore, it can be ambiguous to refer to 'Talairach space' (as it is unclear whether you are referring to the coordinate system or the brain in the Talairach and Tournoux atlas). For clarity, it is helpful to be clear as to the template used for normalization. As noted, the MNI / ICBM template is currently the most popular. For more details see www.mrccbu.cam.ac.uk/Imaging/mnispace.html.
} 


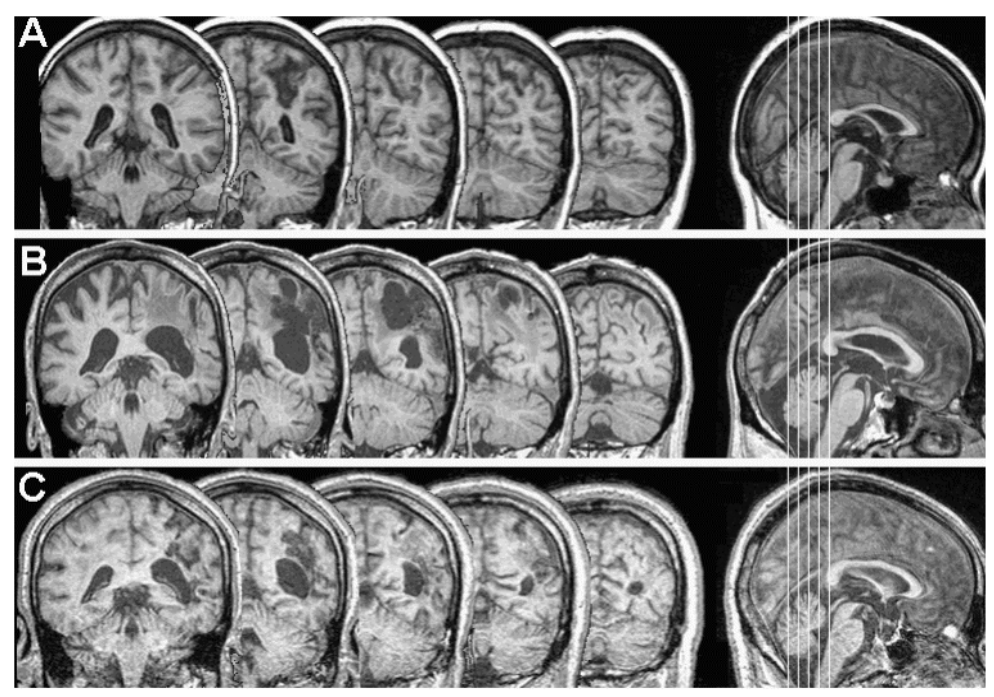

Fig. 3. Normalized T1-weighted MRI scans from three right-hemisphere patients. Note that they all display similar size, shape and orientation. These brains were normalized using SPM99's masking feature as described in the text. These slices correspond to Talairach Y values of -40 , $-48,-56,-64$ and $-72 \mathrm{~mm}$.

closely aligned to each other. A second feature is that normalization preserves the unique features of each brain. This is useful, as one can clearly see the different extent of the lesions. Inherent to the fact that normalization preserves the unique elements of the image is the fact that the normalizations are not 'perfect'. An algorithm that attempted to match all of the sulci would lead to many local distortions and would mask unique features. Further, each individual has a unique sulcal pattern [18]. Indeed, some individuals completely lack some of the secondary and tertiary sulci [13]. Therefore, the concept of a 'perfect' normalization is ambiguous: any normalization will be the result of a series of compromises to preserve the overall size of a region while trying to match the position of major sulci. A good analogy is to think of sulcal patterns as fingerprints - with each individual having a unique set.

The type of normalization that we have described above matches the overall shape, size and orientation of the brain while preserving individual local features. As noted above, the normalization transforms are computed on a spatially smoothed copy of the source image, which reduced fine image detail such as the positions of small sulci. One consequence of this is that Talairach coordinates in images normalized with this sort of algorithm may have only an approximate relationship to the location of individual sulci and to Brodmann's Areas [10,11]. In addition, one could argue that reporting lesion data in terms of Talairach coordinates or sulcal landmarks (as estimated on the individual images) is more appropriate for neuropsycho- logical data than trying to specify the site of the lesion in terms of Brodmann's Areas. Brodmann's Areas are strictly defined by cytoarchitecture, which cannot be directly observed in MRI scans. Based on histological work, Zilles and colleagues [21] have noted that both normalization and sulcal landmarks are not generally precise indicators of the borders of cytoarchitectonic areas.

To summarize, normalization is a technique for coregistering brains to a common template. This allows one to make comparisons across individuals while retaining many of the unique features of each brain (e.g. sulcal patterns). The Talairach coordinate system is used to describe locations in normalized space.

\section{Technical difficulties facing normalization of patient scans}

Despite the clear advantages of presenting patient lesions using normalized MRI scans, this technique remains rare in the neuropsychological literature. In the past, a number of technical obstacles have prevented neuropsychologists from presenting normalized scans. First of all, MRI scans have only recently become available for most neuropsychologists (replacing CT scans where the distance between slices is too large to allow robust normalization). Second, early algorithms for matching damaged patient brains with standard templates were either time consuming or unreliable. Third, 
software for mapping lesion location on MRI scans was expensive, nonexistent, or difficult to use. Finally, a number of proprietary clinical MRI formats exist, and it was difficult to convert these clinical formats to the scientific file formats that are recognized by normalizing software. In this section, each of these hurdles will be described. The next section will describe recent developments that address each of these issues.

In the past, only a small percentage of neurological patients received high quality MRI scans. While CT scans are useful for clinical diagnosis, they are less than ideal for scientists studying brain function. First of all, most CT scans have very large interslice distances, so damage to brain regions that lie between successive slices must be extrapolated. Second, CT scans often do not provide the anatomical and clinical accuracy of combined T1 and T2 MRI scans. Clinical T2-weighted images excel in distinguishing fluids from other materials - specifically cerebral spinal fluids and most pathologic lesions (edema, tumor and infarction). On the other hand, T1-weighted images show a high degree of contrast between gray and white matter, giving excellent anatomical information [12].

Attempting to normalize MRI scans from neurological patients with standard anatomical atlases can be an unreliable procedure. The fundamental problem is that the templates are based on neurologically intact brains, and the region of the lesion can disrupt the normalization (as the lesion intensity is radically different from the corresponding region in the template). One option would be to normalize patient brains by hand - manually adjusting the overall brain size, angle, position, etc. to match the anatomical template. However, manual methods are time consuming (adjustments must be made in all three dimensions) and subjective [15]. As mentioned earlier, normalization is an iterative process, where small adjustments are tested to see if they improve the alignment of the image. One potential problem with this process is that it can get trapped in a "local minimum" - where the algorithm settles for an incorrect set of spatial transformations because small changes in alignment reduce the overall fit even though a bigger change would improve it. By reducing the correspondence of a correct fit, lesions can greatly increase the chance that the normalization functions will settle on an incorrect alignment.

Neuropsychologists have traditionally been slow to present MRI scan data partly because the software to view images and define lesions was expensive, difficult to use and required exotic hardware. Until recently, brain-imaging software required a high-end worksta- tion with a large amount of memory. For example, a single standard high resolution T1 scan $(256 \times 256 \times 128$ voxels, storing 16-bits of data per voxel) can consume $16 \mathrm{MB}$ of hard disc storage, and will require a considerable amount of random access memory for viewing. Few neuropsychologists had access to these systems. In addition, most of the available brain-imaging software was designed for neuroimagers, and these packages did not have many tools to aid neuropsychologists in identifying and measuring the lesion volume.

A final challenge faced by neuropsychologists is that there are many manufacturers of MRI, CT and PET scanner systems. Generally, each of these systems saves images in their own proprietary format. These proprietary clinical formats are different from the scientific formats used by most normalizing software and most neuroimagers. Therefore, the popular scientific tools have been unable to view or modify clinical scans.

\section{Recent advances}

The previous section reviewed the numerous obstacles that have prevented neuropsychologists from normalizing patient scans. This section describes a number of recent technical advances that can aid neuropsychologists hoping to normalize scans from neurological patients. Taken together, these techniques are useful for displaying lesion location in stereotaxic space.

Until recently, high-resolution MRI scans were rare and expensive. Fortunately, MRI scans are becoming more common. This is a trend that will continue in the future. The combination of T2-weighted scans (that generally show precise pathological information) and T1-weighted scans (with their excellent anatomical definition) allow scientists to accurately map the location and extent of lesions. High-resolution T1 scans offer thin slice thickness, so there is minimal need to interpolate the extent of the lesion between slices. With a small voxel size, and a field of view that covers the entire brain, many MRI sequences are ideal for accurate automated normalization.

One danger when normalizing scans from a neurological patient is that the unusual intensity of the lesion can disrupt the normalization. In addition to the abnormality caused by the lesion itself, scans from patients often show greatly dilated ventricles, mass effects and (non-magnetic) aneurysm clip artifacts that can also interfere with the normalization process. In particular, nonlinear transformations that improve normalization of scans from intact individuals can lead to the defor- 
mation of patient lesions. These functions are heavily influenced by local detail, and they can greatly distort damaged regions. For example, a dark-appearing lesion in the white matter can be crushed so that the overall white matter for the patient's scan most closely resembles the template scan of a healthy brain. One option is to only apply linear transforms when normalizing scans from patients. While this somewhat reduces the fit for intact regions, these 'affine only' transforms are fairly robust even when confronted with very large lesions.

Brett, Leff, and Ashburner (www.apnet.com/www/ journal/hbm2000/6786.html) have developed routines for SPM99 that allow accurate normalization of scans from lesioned brains. They describe a simple technique for masking the lesion, so that the unusual signal from the lesion does not influence the computation of the transformations. They demonstrate that this technique can allow both affine and nonlinear transformations to be accurately computed. In addition, this method is straightforward to apply. First, the lesion extent is drawn on top of the scan. This stage allows the user to define the regions of the original scan that will not have equivalent intensities in the template image (e.g. the lesion, clip artifacts, etc.). Next, this 'region of interest' (ROI) is inverted, so that regions outside the abnormal tissue have values of 1 , and the abnormal tissue contains zeros. Next the inverted ROI is smoothed using the same function that will be applied to the brain image during normalization, and finally thresholded so that values in the smoothed image that are not very close to 1 are set to zero. This image is the 'source mask'. The reason for smoothing and thresholding the ROI is to deal with the fact that the source brain image is smoothed (prior to normalization), and therefore regions of abnormal image intensity will 'bleed' into surrounding voxels. Smoothing and thresholding the ROI ensures that the normalization will only be conducted on healthy tissue with a contribution from abnormal tissue that is very near zero. Next, one conducts an SPM normalization using the source mask to define the area of the image that is to be used to compute the transforms. Specifically, when the normalization is testing a transformation, it will calculate the pixel values for the source image after the transformation, and also the matching pixel values from the mask image. Wherever the pixel values for the mask image are 0 , the program sets the cost function (the squared difference between the transformed source image and the template image for that pixel) to 0 . Using this masked cost function, the program can calculate the optimum linear and nonlinear transforms that match only the regions that fall outside of the source mask (i.e. based only on the healthy tissue). Finally, the transforms are applied to the entire brain image (for example, if the brain needed to be 'zoomed' to be $20 \%$ smaller, both the lesion and the healthy tissue would both be scaled appropriately). This 'lesion masking' technique ensures that the lesion normalization is based solely on the portions of the source image that match the template image. Brett and colleagues have demonstrated that this technique can greatly improve the normalization accuracy for images from stroke patients. However, there are some limitations to the lesion masking technique. If the lesion is very large, and especially if the abnormal area is large and bilateral, then the normalization may have too little information to work on. In this case, the program may calculate transformations that match the remaining brain very well, but cause severe distortions in the masked area, that are clearly incorrect, and are obvious to visual inspection. In the evaluations of Brett et al., the technique worked well even for lesions that included most of one hemisphere. If the lesion is large enough to cause problems for the lesion masking technique, then one may need to use an affine-only or manual normalization.

In the past, software for tracing lesions was expensive, generally difficult to use and required exotic hardware. For example, Brainvox brain imaging software [6] requires a SGI workstation. A fundamental problem has been that brain images contain a large amount of data - and manipulating this vast amount of data required large hard disk drives, fast CPUs and large amounts of RAM. Previously, these hardware requirements were only fulfilled by the high-end workstations. However, cheap desktop computers have recently become much more powerful, and modern desktops are now more powerful than the previous generation of workstations.

A second problem is that the number of neuropsychologists has been too small to justify software companies investing the money to develop software for this market. A recent solution is MRIcro, a free software package released in January 1999. MRIcro is both fairly ease to use and has a number of tools to help neuropsychologists. MRIcro will run on any modern Windows or Linux Intel-compatible PC. The software uses a graphical user interface that makes it easy to use. It should be stressed that MRIcro was not designed for clinical use. However, the software has found a niche being used by scientists working with medical images. One can use MRIcro to rapidly 
Table 1

This table lists free tools for converting proprietary medical image formats to the Analyze format used by scientific normalization and viewing software. In addition, the table lists the URLs for downloading these packages

\begin{tabular}{lll}
\hline Tool & System & Formats read / URL \\
\hline MRIcro & Windows, Linux & - Analyze, DICOM, NEMA, GE LX, GE Genesis, Interfile, \\
& & ECAT6/7, Siemens Magnetom Vision, Picker CT, SPMwin, Raw \\
& & - www.psychology.nottingham.ac.uk/staff/cr1/mricro.html \\
ImageJ & Windows, Unix, Macintosh & - Analyze, BMP, DICOM, GIF, JPEG, TIFF \\
& & - /rsb.info.nih.gov/ij/ \\
MedCon & Windows, Unix & - Analyze, DICOM, NEMA, ECAT6, GIF \\
& & - petaxp.rug.ac.be/nolf/ \\
ImageConverter & Windows & - Analyze, Siemens System 7, Shimadzu HeadTome IV, Hamamatsu \\
& & Photonics SHR2000, GEMS 2048-15B \\
& & - www.fil.ion.bpmf.ac.uk/spm/spmw.html \\
\hline
\end{tabular}

view a scan and define the damaged region. Modern computers running Windows or Linux can also use $\mathrm{SPM}^{2}$ and take advantage of its advanced normalization features. MRIcro can be downloaded from the web: www.psychology.nottingham.ac.uk/staff/cr1/ mritut.htm.

Both MRIcro and SPM use graphical interfaces. With MRIcro, many of the standard functions are similar to the functions of other Windows applications (see Fig. 4). For example, to load an MRI scan you can simply drag and drop its icon onto the program (MRIcro will automatically identify the image format, regardless of whether it is in Analyze, DICOM, or a supported proprietary format). The application includes drawing tools that allow the user to quickly outline and save the lesion location. Creating these regions of interest (ROIs) allows the user to compute lesion volume, compare regions of mutual involvement and create lesion masks for SPM99 (the technique developed by Brett et al. described earlier). Performing an accurate normalization of a patient scan is somewhat more involved. However, a step-by-step tutorial for using MRIcro and SPM to normalize and present scans is available on the web (www.psychology.nottingham.ac.uk/staff/cr1/ mritut.htm). Following this guide, most users can quickly learn how to display lesions in stereotaxic space. Additional tips are available from the Medical Research Council's Cognition and Brain Sciences Unit in Cambridge (www.mrc-cbu.cam.ac.uk/Imaging/). In sum, the introduction of modern, more powerful com-

\footnotetext{
${ }^{2}$ While SPM99 is free, it does require a Matlab license. One alternative is to use the freeware "SPM for Windows" (a.k.a. SPMwin) a free implementation of SPM96 that does not require MatLab. One limitation with SPMwin is that it cannot perform SPM99's lesion masking (as outlined in this article). Therefore, when normalizing images of lesioned brains, SPMwin users should conduct affine-only transformations. To find out more about SPM or SPMwin, visit www.fil.ion.bpmf.ac.uk/spm/spmw.html.
}

puters and the advent of free brain imaging software allows neuropsychologists to work with MRI scans using their desktop computer.

The final hurdle confronting neuropsychologists was the large number of proprietary medical image formats. Many scanner manufacturers have designed their own image formats. This makes the user dependent on the manufacturers' software for viewing and printing scans. Unfortunately, the scientific software available for normalizing images cannot read these formats. However, there has recently been a major shift in the industry with most new scanners supporting the DICOM format (or its predecessor, the NEMA format). Therefore, most neuropsychologists will only require software to convert images from the DICOM standard to a scientific standard. In addition, a number of free software packages have been developed that can convert a broad range of medical image formats into a standard scientific format (the 'Analyze' format). Table 1 lists a number of free tools for converting medical images. For example, MRIcro can convert the most common clinical images to Analyze format.

\section{Conclusions}

Neuropsychological research can play an important role in the developing field of cognitive neuroscience. Lesion studies can provide convergent information and help guide studies using different methods. However, one traditional weakness with lesion studies has concerned the presentation of anatomical data. Presenting anatomical data in a consistent framework can improve comparisons across studies and improve the correspondence of lesion studies with other methods. This article describes the methods used by neuroimagers and suggests that it is a robust tool for lesion studies. This method can improve our understanding of the anatomical basis of neurological syndromes. 


\section{References}

[1] J. Ashburner and K.J. Friston, Nonlinear spatial normalization using basis functions, Human Brain Mapping 7 (1999), 254 266.

[2] D.L. Collins, P. Neelin, T.M. Peters and A.C. Evans, Automatic 3D intersubject registration of MR volumetric data in standardized Talairach space, Journal of Computer Assisted Tomography 18 (1994), 192-205.

[3] H. Damasio and A.R. Damasio, Lesion Analysis in Neuropsychology, New Yourk, Oxford, 1989.

[4] E. De Renzi, Prosopagnosia, in: Patient-based approaches to cognitive neuroscience, M.J.F.a.T.E. Feinberg, ed., MIT Press, Cambridge, MA, 2000.

[5] A.C. Evans, M. Kamber, D.L. Collins and D. Macdonald, An MRI-based probabilistic atlas of neuroanatomy, in: Magnetic Resonance Scanning and Epilepsy, S.S. Shorvon, D. Fish, F. Andermann and G.M.H. Bydder, eds, 1994.

[6] R.J. Frank, H. Damasio and T.J. Grabowski, Brainvox: an interactive multimodal visualization and analysis system for neuroanatomical imaging, NeuroImage 5 (1997), 13-30.

[7] R. Frey, D.L. Woods, R.T. Knight and D. Scabini, Defining functional cortical areas with "averaged" CT scans, Society of Neuroscience Abstracts 13 (1987), 1266-1267.

[8] K.J. Friston, J. Ashburner, C.D. Frith, J.B. Poline, J.D. Heather and R.S.J. Frackowiak, Spatial registration and normalization of images, Human Brain Mapping 3 (1995), 165-189.

[9] N. Kanwisher, J. McDermott and M. Chun, The fusiform face area: A module in human extrastriate cortex specialized for face perception, Journal of Neuroscience 11 (1997), 43024311.

[10] J.L. Lancaster et al., Automated Talairach atlas labels for functional brain imaging, Human Brain Mapping 10 (2000), 120 131.

[11] J.C. Mazziotta, A.W. Toga, A. Evans, J.L. Lancaster and P.T.
Fox, A probabilistic atlas of the human brain: Theory and rational for its development, Neuroimage (1995), 89-101.

[12] P.G. Morris, Nuclear magnetic resonance imaging in medicine and biology, Claredon Press, Oxford, 1986.

[13] M. Ono, S. Kubik and C.D. Abernathey, Atlas of the cerebral sulci, Theime Medical Publishers, New York, 1990.

[14] C.J. Price, D. Howard, K. Patterson, E.A. Warburton, F.J. Friston and R.S.J. Frackowiak, A functional neuroimaging description of two deep dyslexic patients, Journal of Cognitive Neuroscience 10 (1998), 303-315.

[15] M. Sugiura et al., Anatomic validation of spatial normalization methods for PET, Journal of Nuclear Medicine 40 (1999), 317-322.

[16] J. Talairach and P. Tournoux, Co-planar Stereotaxic Atlas of the Human Brain, George Thieme, New York, 1988.

[17] P.M. Thompson, M.S. Mega, K.L. Narr, E.R. Sowell, R.E. Blanton and A.W. Toga, Brain image analysis and atlas construction, in: Handbook of Medical Imaging, M.S.a.J.M. Fitzpatrick, ed., SPIE Press, Bellingham, WA, 2000.

[18] P.M. Thompson, C. Schwartz, R.T. Lin, A.A. Khan and A.W. Toga, 3D Statistical Analysis of Sulcal Variability in the Human Brain, Journal of Neuroscience 16 (1996), 4261-4274.

[19] D.J. Werring, A.T. Toosy, C.A. Clark, G.J.M. Parker, G.J. Barker, D.H. Miller and A.J. Thompson, Diffusion tensor imaging can detect and quantify corticospinal tract degeneration after stroke, Journal of Neurology Neurosurgery and Psychiatry 69 (2000), 269-272.

[20] R.P. Woods, S.R. Cherry and J.C. Mazziotta, Rapid automated algorithm for aligning and reslicing PET images, Journal of Computer Assisted Tomography 16 (1992), 620-633.

[21] K. Zilles et al., Quantitative analysis of sulci in the human cerebral cortex: Development, regional heterogeneity, gender difference, asymmetry, intersubject variability, and cortical architecture, Human Brain Mapping 5 (1997), 218-221. 


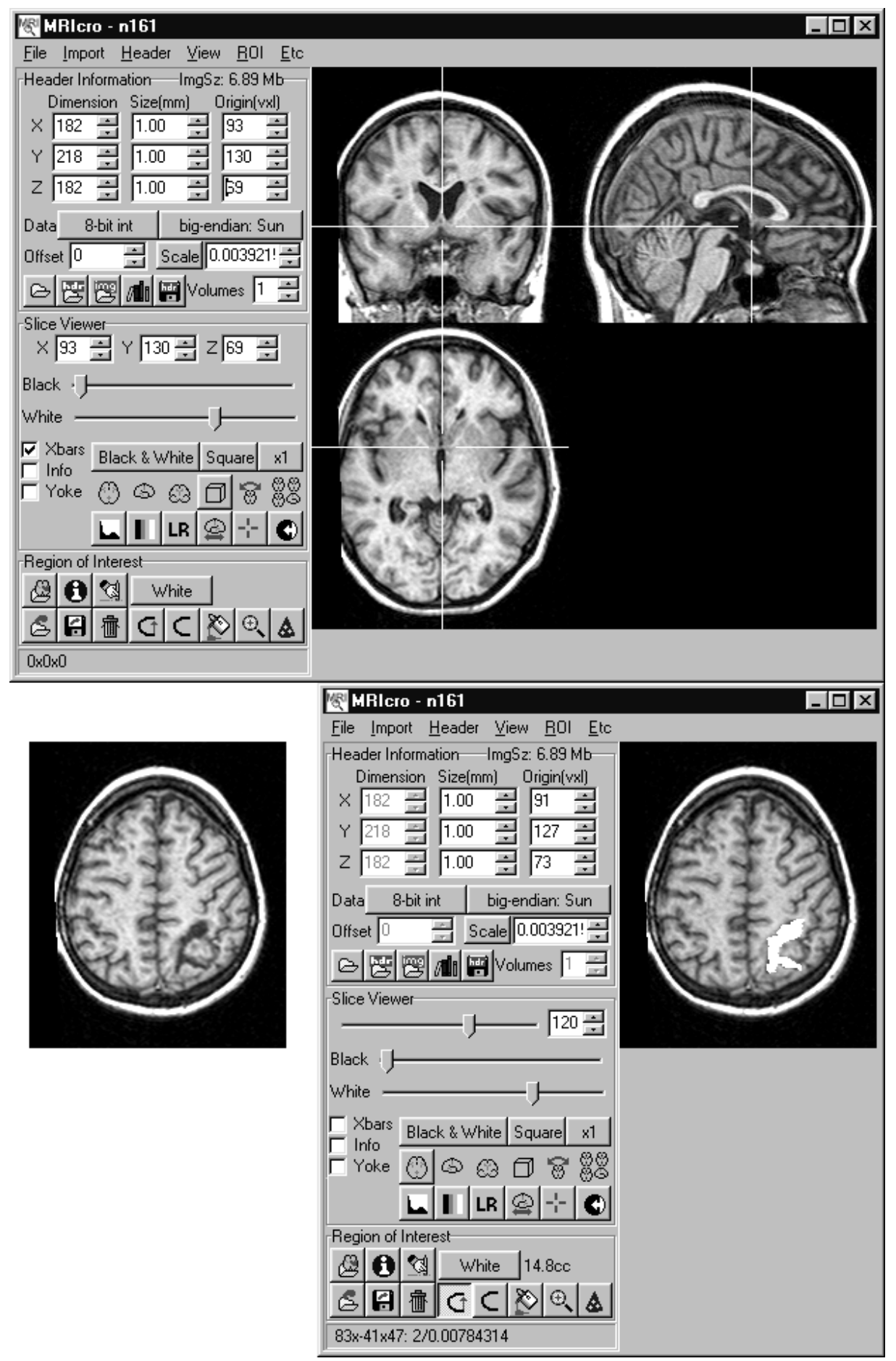

Fig. 4. MRIcro is a free application that can display images from a broad range of scanners. It includes drawing tools that allow neuropsychologists to compute lesion volume, identify regions of mutual involvement, and create lesion masks for SPM99. The top panel illustrates the approximate location of the anterior commissure, the landmark used as the origin for Talairach space. The lower left panel shows an axial slice where a lesion can be observed. The bottom right panel shows how the user can draw a region of interest (ROI) that includes the lesion - depicted as white. The volume of the ROI is reported in the lower-left portion of the MRIcro window (here, 14.8cc). Also, note that the pen tool has been selected (as signified by the depressed-button depicting a curved-line visible in the lower left of the window), allowing the user to outline the ROI. 


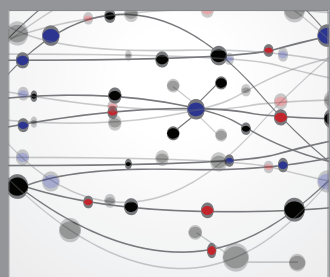

The Scientific World Journal
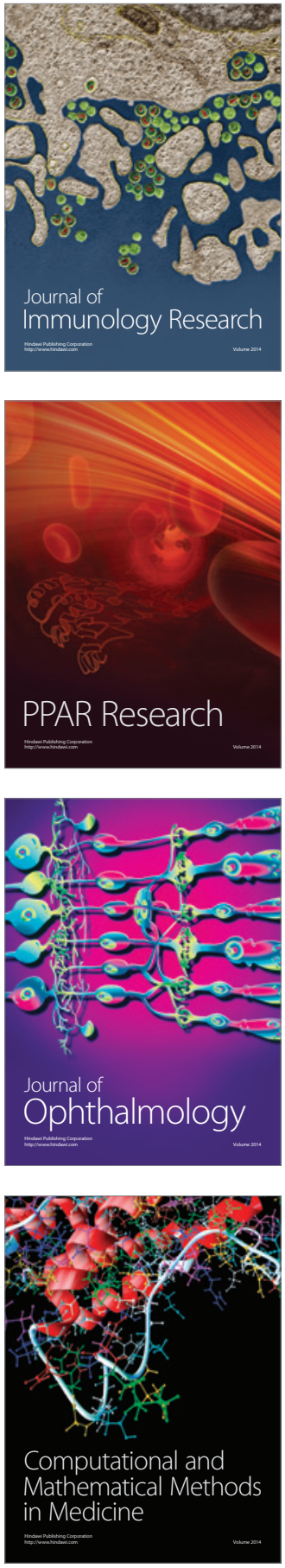

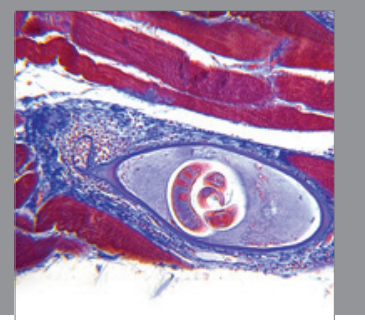

Gastroenterology

Research and Practice
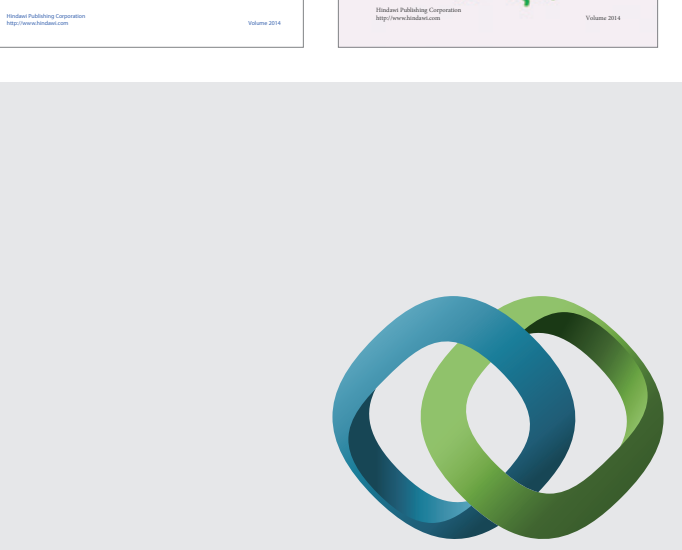

\section{Hindawi}

Submit your manuscripts at

http://www.hindawi.com
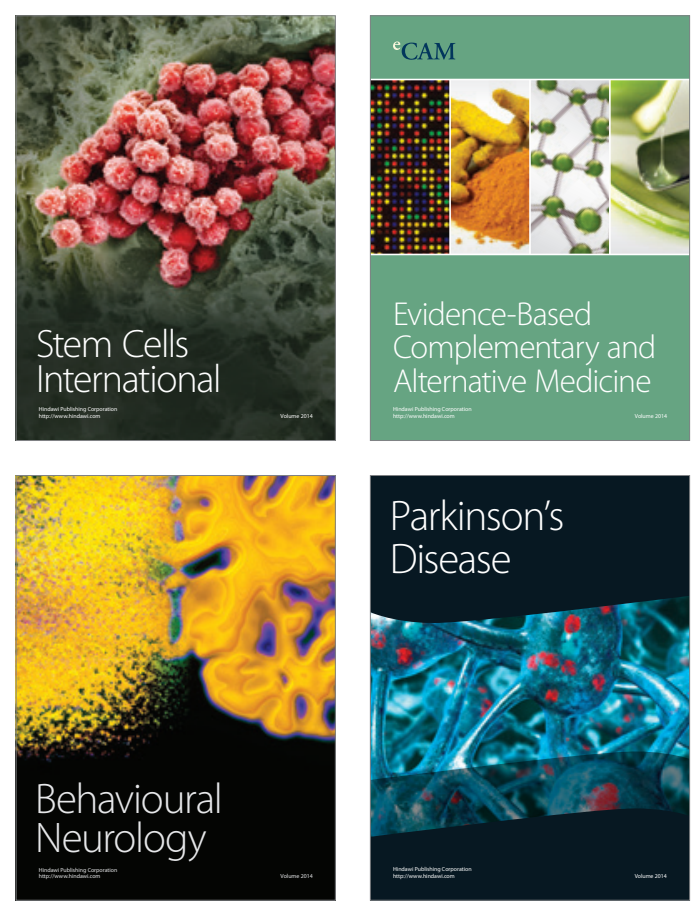

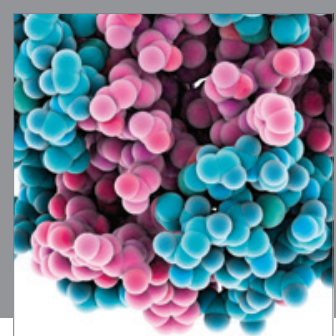

Journal of
Diabetes Research

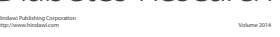

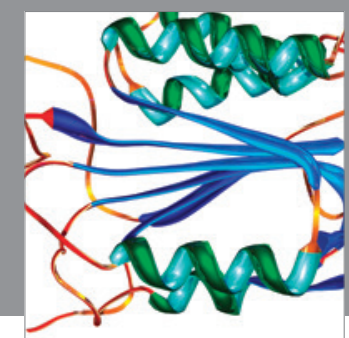

Disease Markers
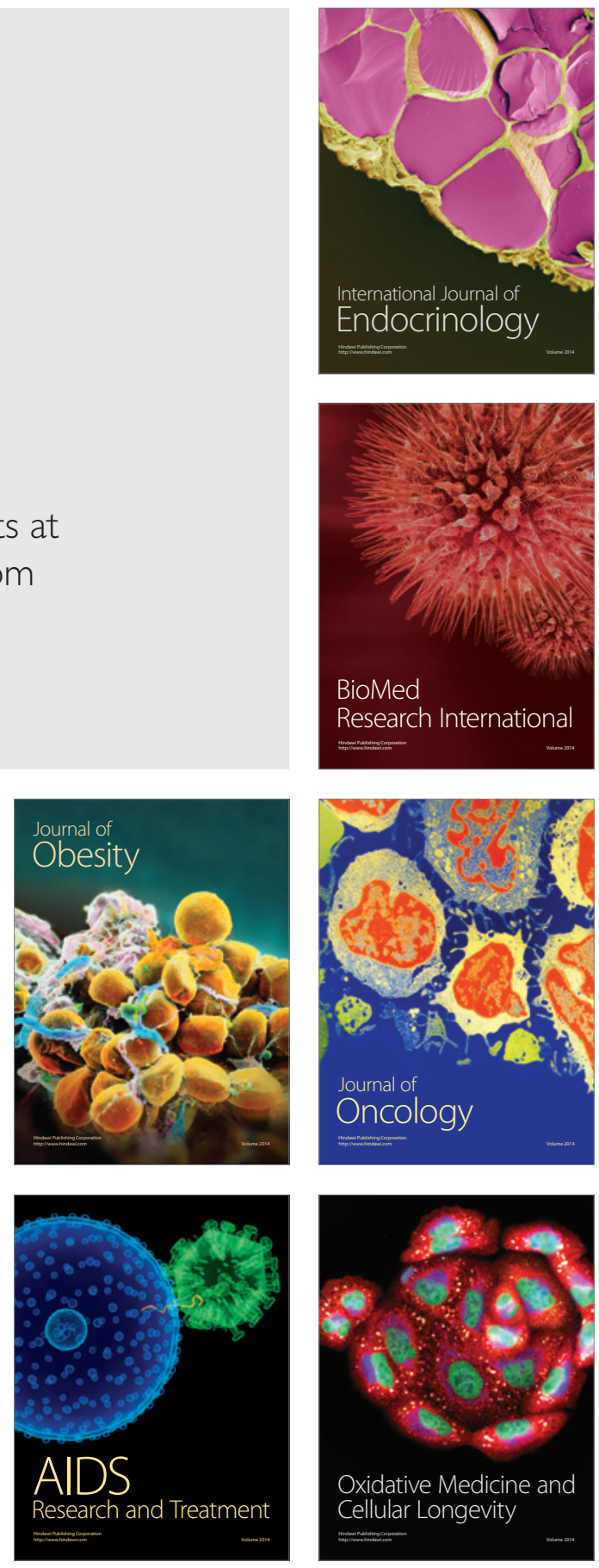